\title{
Electronic structure of overstretched DNA
}

\section{Citation}

Maragakis, Paul, Ryan Lee Barnett, Efthimios Kaxiras, Marcus Elstner, and Thomas Frauenheim. 2002. "Electronic Structure of Overstretched DNA." Physical Review B 66 (24). https:// doi.org/10.1103/physrevb.66.241104.

\section{Permanent link}

http://nrs.harvard.edu/urn-3:HUL.InstRepos:41384082

\section{Terms of Use}

This article was downloaded from Harvard University's DASH repository, and is made available under the terms and conditions applicable to Other Posted Material, as set forth at http:// nrs.harvard.edu/urn-3:HUL.InstRepos:dash.current.terms-of-use\#LAA

\section{Share Your Story}

The Harvard community has made this article openly available.

Please share how this access benefits you. Submit a story.

\section{Accessibility}




\title{
Electronic structure of overstretched DNA
}

\author{
Paul Maragakis, ${ }^{1}$ Ryan Lee Barnett, ${ }^{1}$ Efthimios Kaxiras, ${ }^{1}$ Marcus Elstner,,${ }^{2,3}$ and Thomas Frauenheim ${ }^{2}$ \\ ${ }^{1}$ Physics Department and Division of Engineering and Applied Sciences, Harvard University, 02138 Cambridge MA \\ ${ }^{2}$ University of Paderborn, Fachbereich Physics, D-33098 Paderborn, Germany. \\ ${ }^{3}$ German Cancer Research Center, Department of Molecular Biophysics, D-69120 Heidelberg, Germany.
}

\begin{abstract}
Minuscule molecular forces can transform DNA into a structure that is elongated by more than half its original length. We demonstrate that this pronounced conformational transition is of relevance to ongoing experimental and theoretical efforts to characterize the conducting properties of DNA wires. We present quantum mechanical calculations for acidic, dry, poly $(\mathrm{CG}) \cdot \operatorname{poly}(\mathrm{CG}) \mathrm{DNA}$ which has undergone elongation of up to $90 \%$ relative to its natural length, along with a method for visualizing the effects of stretching on the electronic eigenstates. We find that overstretching leads to a drastic drop of the hopping matrix elements between localized occupied electronic states suggesting a dramatic decrease in the conductivity through holes.
\end{abstract}

PACS numbers: 87.14.Gg, 72.80.Le, 87.15.Aa

The possibility of efficient charge transfer along the stacked $\pi$ orbitals in DNA was proposed 40 years ago [1]. In recent years its conducting properties were tested in several contexts: DNA has been used as a template on top of which nanowires are built 2 and there is hope that self-assembled DNA junctions between conductors will function soon. There has also been renewed interest in building and characterizing molecular wires of plain DNA

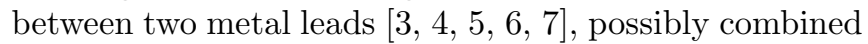
with changes of the major constituents of the molecule to improve its properties [8. There is ongoing debate as to whether DNA is conducting, semi-conducting, insulating or all of the above [9]. There are several reasons for this controversy. There is great diversity of the DNA forms in terms of its composition, length, and structure. Counterions and impurities that can attach to the phosphate groups and the grooves of the molecule need to be taken into account. Finally, environmental factors such as temperature and contact resistance can play a major role.

Experiments done 50 years ago by Wilkins et al. [10], suggested that overstretched DNA (that is, substantially longer than its natural length) undergoes a transition to a structure that can accommodate elongation up to twice the length of relaxed DNA. A major breakthrough in understanding the mechanical properties of DNA was achieved through single molecule stretching experiments [11, 12, 13]. It was shown that by gradually increasing the axial stretching force $F$ along the DNA helix, the molecule first uncoils, then, at $F 5 \mathrm{pN}$, it reaches its natural length, and then exhibits a stiff elastic response for forces of up to $50 \mathrm{pN}$. At $F 50$ to $80 \mathrm{pN}$, DNA undergoes a pronounced and abrupt structural transformation to a yet unknown structure that is elongated by more than $50 \%$. Further increase in force leads to breaking after it has stretched to twice its natural length. In this letter we argue that these overstretched forms of DNA are relevant in the quest for novel device components, we develop a visualization method for understanding the nature of electronic states in these forms, and we present the first detailed discussion of their electronic properties.

We use an efficient quantum mechanical electronic structure method 14 that treats all the valence electrons and that accounts for the charge transfer involved in biological molecules and semiconducting surfaces. This approach, called self-consistent-charge density-functional tight-binding method, is based on an expansion of the Kohn-Sham energy functional [15, 16] in terms of the charge density. The zeroth order term results in a tightbinding Hamiltonian, and the second order term incorporates the charge redistribution. The method is able to deal with the very large numbers of atoms involved in biologically relevant systems. As a test, we performed calculations on a periodic homopolymer of $\operatorname{poly}(\mathrm{C}) \cdot \operatorname{poly}(\mathrm{G})$ with 10 base pairs per turn and reproduced the results of dePablo et al. [5], which to our knowledge is the only $a b$ initio electronic structure calculation of a periodic DNA structure [17. Our results are in excellent agreement to their calculations: we find a $2.1 \mathrm{eV}$ bandgap between the highest occupied and lowest unoccupied eigenstates, compared to $2.0 \mathrm{eV}$ reported in Ref. [5]. The wavefunctions of the highest occupied valence band are located on the guanines while the wavefunctions of the lowest unoccupied band are located on the cytosines, also in agreement with Ref. [5].

For the purpose of the present study, calculations were performed on a variety of DNA polymers with different compositions employing both infinite periodic and finite structures. We limit our discussion to $\operatorname{poly}(\mathrm{CG}) \cdot \operatorname{poly}(\mathrm{CG})$ as a representative structure for which well established overstretched configurations have been published 18. Our results on finite DNA polymers show the existence of states localized at the opposite ends of the molecule. The energy levels of these states lie between the regular bands that appear in the periodic structures. We removed these states before analyzing the electronic properties, and will refer to the remaining states as the "restricted band structure" of the non-periodic 
configuration. As a test, we compared 18 base pair restricted band sturcture with periodic structures with 10 base pairs per unit cell and found the band structures virtually indistinguishable. A boundary region of 3 basepair layers at each end of the molecule was sufficient to contain the spurious end states. Fig. 1 displays the band structure of a periodic sequence with a unit cell consisting of 10 base pairs. The sharp features of the density of states are due to the one dimensional nature of DNA. In all periodic sequences there is an extra symmetry, which for the poly $(\mathrm{CG}) \cdot \operatorname{poly}(\mathrm{CG})$ structure is the translation by two base pairs along the stack, together with a rotation of 72 degrees around the helical axis: this symmetry is generated by the screw operator that is isomorphic to a pure translation operator. Structures obtained by short (50 ps) molecular dynamics runs at $50 \mathrm{~K}$ using classical force fields [19], indicate that minor deformations of the ideal configuration destroy the sharp features in the electronic structure.

We now move on to a discussion of overstretched DNA. First we elaborate on how this is relevant in the construction of DNA nanowires. One way to attach DNA molecules to substrates is the technique of "molecular combing" 20]. This method uses the meniscus forces that develop between the solution surface and the device surface template to uncoil the DNA and stretch it between two electrodes as the device is pulled out of the solution. A description of recent experiments estimates that the forces that are exerted on the DNA molecules are of order $100 \mathrm{pN}$ [21, more than enough to stretch them past their natural length. This is indicated by recently built devices [22 where the distance between the electrodes was about 30\% longer than the natural length of the DNA molecules. The overstretching can have a variety of consequences on the electronic states.

Elongated DNA structures have been determined in the pioneering study of Lebrun and Lavery [18, which model the adiabatic elongation of selected DNA molecules. There are two distinct modes of overstretching corresponding to pulling the opposite 3'-3' ends and 5'-5' ends. The former mode leads to DNA unwinding, consistent with a recent estimate of the helicity of overstretched DNA [23], whereas the latter leads to a contraction of the diameter of the helix. Both overstretching modes can accommodate elongations of up to $90 \%$ without breaking the molecule.

We next present the inherent electronic states of the overstretched structures and then consider electron transport aspects. For all the calculations we used acidic, dry, 18 base-pair strings of DNA, and we performed the projection of the end states in the manner stated before. The quasi one-dimensional structure of all the forms leads to the formation of clear minibands in the energy spectrum, similar to those shown in Fig. 1. The band structure of the overstretched forms have an overall resemblance to the band structure of the original unstretched

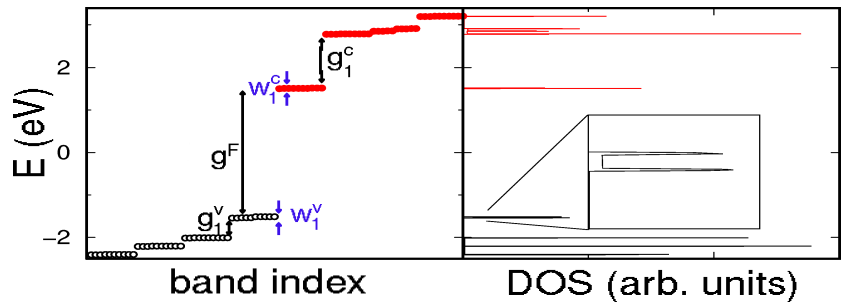

FIG. 1: The band structure around the Fermi energy (left) with the corresponding density of states (right) for the periodic poly $(\mathrm{CG}) \cdot \operatorname{poly}(\mathrm{CG})$ form. The inset shows a detail of the density of states at the highest occupied valence band. We denote the bandgap around the Fermi energy $\left(g^{F}\right)$, the widths of the highest valence $\left(\mathrm{w}_{1}^{v}\right)$ and lowest conduction $\left(\mathrm{w}_{1}^{c}\right)$ minibands, and the gaps between these and the neighboring minibands $\left(\mathrm{g}_{1}^{v}\right.$ and $\left.\mathrm{g}_{1}^{c}\right)$.

form, and many of the minibands near the Fermi energy can be continuously mapped to their counterpart in the unstretched configuration. This is mostly because of the molecular structure of the overstretched forms as will become clear in the following discussion. For some of the most deformed structures, especially the $90 \%$ overstretching in the 5' -5 ' mode, several of the minibands are mixed and small but clear gaps present in less deformed structures disappear.

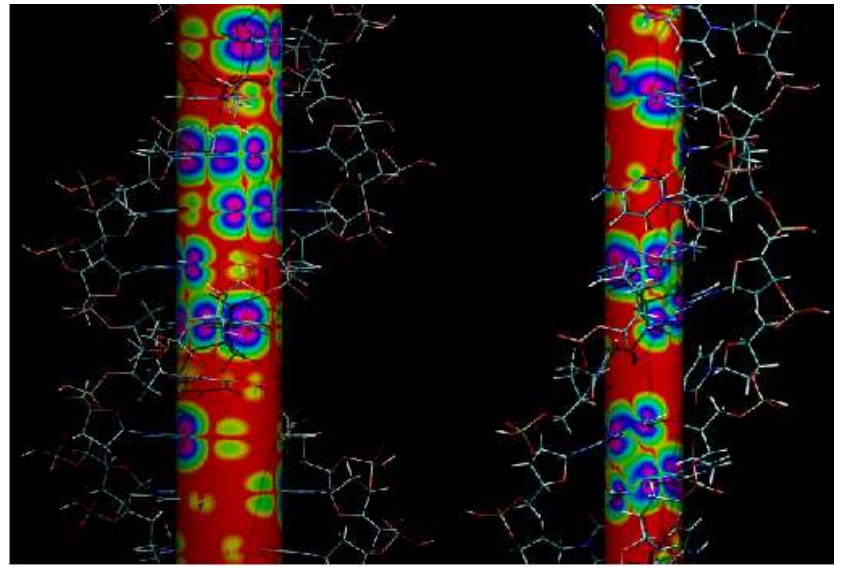

FIG. 2: The left panel shows a piece of poly $(\mathrm{CG}) \cdot \operatorname{poly}(\mathrm{CG})$ B-DNA. Along its helical axis is a cylinder on which we plot the contours of the valence band electron density. The right panel shows the same region for a $30 \%$ stretched structure.

We have devised a new scheme for visualizing the electronic states of DNA, which naturally accounts for the symmetry of this molecule. The basic concept of this scheme is to unfold contour plots of the band electron density evaluated at cylindrical surfaces centered on the helical axis, as shown in Fig. 2. In this figure we show the structure at its natural length together with one that is elongated by $30 \%$ in the 3'-3' overstretching mode. With the help of these plots we can understand most of the band structure features of the stretched forms. The elon- 
gation to the overstretched form is achieved by changing the dihedral angle configuration of the backbone of the molecule, thus leaving the local part of the orbitals essentially intact.

In Fig. 3 (a) we present the valence band electron density of selected stretched poly $(\mathrm{CG}) \cdot \operatorname{poly}(\mathrm{CG})$ structures using our visualization scheme. We choose the radii of the cylinders to follow the atom with the largest electron density for the band that we present. In the unstretched structure, the $\pi$-band is along the guanines. Upon elongation, orbitals from the picture frame are removed while the remaining ones rotate partially as dictated by the new orientation of base pairs. The poly $(\mathrm{CG}) \cdot \operatorname{poly}(\mathrm{CG})$ structure contains pairs of guanines that are closer to each other. These guanines display an overlap of the electronic densities as seen in the top left frame of Fig. 3 (b). Elongation in the 3'-3' mode keeps such paired guanines close to each other which is reflected in the overlap in their electronic densities; the overlap of the electronic densities between guanines from different pairs decreases with elongation. In Fig. 3 (b) we show the conduction band electron density. Note again how the orbitals rotate as the structure is being overstretched, following the rotation of the bases. Note that in the extreme $90 \%$ stretching case, the orbitals become perpendicular to the helical axis.

The changes in the structural features should be of profound consequence to any model dealing with electron transfer since, to a first approximation, the overlap matrix elements depend exponentially on the distance between orbitals. Because of the $\pi$ character of the states near the Fermi level, a non-parallel alignment will strongly affect the values of the overlap integrals. In order to quantify these effects, we considered the nearly degenerate eigenstates in the first valence miniband. We used the linear combination of these states that gives normalized, orthogonal states $\left|\Psi_{i}\right\rangle$, which are maximally localized along the helical axis within the subspace spanned by the delocalized states. The resulting valence states are localized on guanine bases. With these states, we calculated the hopping matrix elements:

$$
t_{i j}=-\frac{1}{2}\left\langle\Psi_{i}\left|\nabla^{2}\right| \Psi_{j}\right\rangle,
$$

between nearest-neighbor sites. Note that for the poly $(\mathrm{CG}) \cdot \operatorname{poly}(\mathrm{CG})$ structure every guanine has 2 guanine neighbors, one above and one below as indicated in Fig. 3, separated by distances of $5.3 \AA$ and $4.2 \AA$. The larger of the two matrix elements corresponds to hopping between sites that are close together; its value remains roughly constant for the $30 \%$ overstretched structures. The smaller of the two matrix elements, which corresponds to sites that are further apart, is dramatically reduced for all the overstretched forms. Since the two different hopping terms are connected in a 1-D series the smallest term will determine the bottleneck for electron transport. The absolute square values of these small hopping matrix elements between these neighbors are given in Fig. A (a). We infer from this figure that if the conduction mechanism is through holes there should be a dramatic drop in conductivity with $30 \%$ overstretching, which in the 3'-3' mode drops further with elongation. In Fig. A (b) we give the hopping matrix elements between states in the conduction band. In the cases where the pairing between nearest neighbor pairs have very different matrix elements we only list the one that would be the bottleneck for this channel. Due to the stronger localization of the conduction band and because of the rather large distance between the occupied sites, the hopping matrix elements are substantially smaller than the ones between the valence states in the structure of natural length. In fact, it is the site that is two bases away which has the strongest overlap in this structure: the C6 atoms between these bases are actually closer to each other $(9.0$ $\AA$ ) than the $\mathrm{C} 6$ atoms between consecutive base pairs (10.0 versus $10.3 \AA$ ). The first and second neighbor hopping terms are indicated in Fig. 3 (b). Since these terms are connected in parallel, this time it is the maximum of the two that will play the dominant role in the conduction mechanism and it is this one that is included in Fig. A (b). We note that if conduction takes place by electrons the conductivity drops with overstretching in the 3'-3' mode but not necessarily in the 5'-5' mode.

The concept of overstretched DNA in relation to electronic device components appears relevant to a variety of experimental and theoretical issues. Experimentally it is possible, and sometimes even unavoidable, to create overstretched DNA molecules. The window of allowable forces that can be applied to stretch DNA to its natural length, but not overstretch, is small (from $5 \mathrm{pN}$ to $50 \mathrm{pN}$ ) compared to the forces applied during molecular combing. Another important aspect is owed to the nature of the extreme stretched forms, it seems possible to expose the bases to the exterior of the molecule allowing impurities to enter the crucial base-pair stack region; this could result in controlling the electronic properties with doping. This might also enable direct visualization of parts of the electronic wavefunctions which was is not possible in the natural DNA forms because of the insulating backbone layer. On the theoretical level, the major existing models (superexchange [24], hopping [24], and polaron [25]), of pure DNA conductivity will be drastically affected by the consequences of overstretching, including changes in the hopping matrix elements and in the low-energy vibrational modes. For long fibers of homogeneous DNA (either a homopolymer, or a short repeated sequence) we expect stretching to appear homogeneously. Regions of varying richness in AT or GC mixture would induce different lengthenings at different parts of the molecule, permitting conduction mechanisms to change along the molecule.

We thank Leo Kouwenhoven and Nina Markovic for 

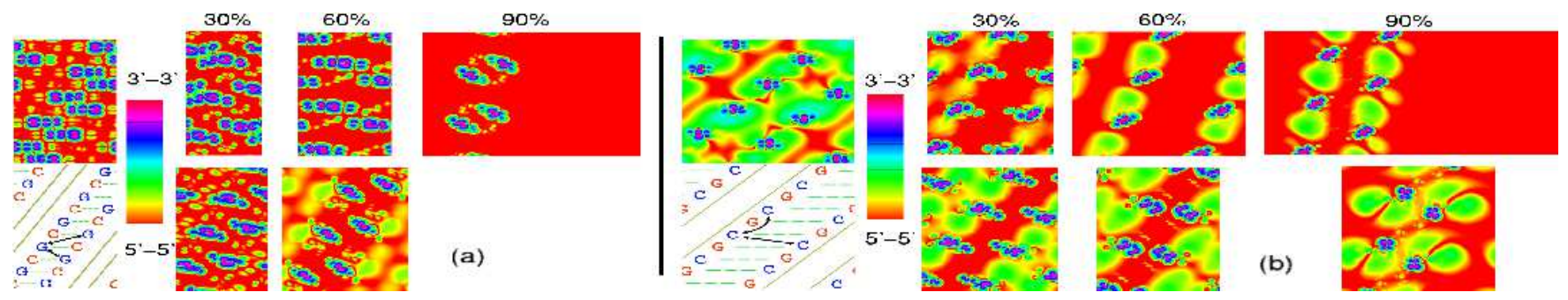

FIG. 3: Cylindrical contour map of the highest valence (a) and the lowest conduction (b) band electron density, for the poly (CG)·poly(CG) DNA (top left) and the 30\%, 60\%, and 90\% overstretched forms (right). The bottom left panel of each figure shows a drawing of the CG base pairs along the cylinder; the arrows indicate bases with the largest couplings. In Fig. (a) the $90 \%$ overstretched 5'-5' form is not shown because the highest valence band mixes with the lower bands. The vertical axis covers a fixed region corresponding to 10 base pairs in the unstretched form. The horizontal axis runs from 0 to $2 \pi$. The radius of the cylinder follows the guanine C5 atom in (a), and the cytosine C6 in Fig. (b). The cylinder surface unit element is kept fixed: this results in a variation of the length of the horizontal axis, proportional to the cylinder radius. The color coding (shown in the color bar between the unstretched and stretched graphs) is fixed between different structures and covers 5 orders of magnitude.

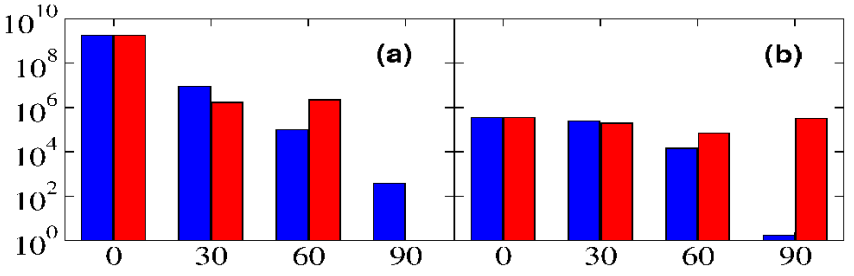

FIG. 4: The values of $\left|t_{i j}\right|^{2}$ from Eq. (1) for (a) the valence and (b) the conduction band. The values are given $\mathrm{eV}^{2}$ multiplied by a factor of $10^{12}$ to facilitate relative comparisons, for overstretching of $0,30,60,90 \%$ relative to the natural length. The blue (left) column at each entry corresponds to the 3'-3' stretching mode and the red (right) column to the 5'-5' mode. In Fig. (a), the entry at the $90 \%$ stretching of the 5'-5' mode is missing because of the mixing the valence band with lower bands.

sharing their experimental insight with us. We are grateful to Richard Lavery for providing the overstretched structures. We thank Hatem Mehrez for his critical reading of the manuscript. This work was supported in part by Harvard's Materials Research Science and Engineering Center, which is funded by the National Science Foundation. This work was partially supported by National Computational Science Alliance under MCB010005N and utilized the Boston University Scientific Computing and Visualization facilities. RLB was supported by a national science foundation graduate research fellowship.

[1] D. D. Eley and D. I. Spivey, Trans. Faraday Soc. 58, 411 (1961).

[2] E. Braun, Y. Eichen, U. Sivan, and G. Ben-Yoseph, Nature 391, 775 (1998).
[3] H.-W. Fink and C. Schonenberger, Nature 398, 407 (1999).

[4] D. Porath, A. Bezryadin, S. De Vries, and C. Dekker, Nature 403, 635 (2000).

[5] P. de Pablo et al., Phys. Rev. Lett. 85, 4992 (2000).

[6] A. Kasumov et al., Science 291, 280 (2001).

[7] K.-H. Yoo et al., Phys. Rev. Lett. 87, 198102 (2001).

[8] A. Rakitin et al., Phys. Rev. Lett. 86, 3670 (2001).

[9] H. W. Fink, Cell. Mol. Life Sci. 58, 1 (2001).

[10] M. H. F. Wilkins, R. G. Gosling, and W. E. Seeds, Nature 167, 759 (1951).

[11] S. B. Smith, Y. J. Cui, and C. Bustamante, Science 271, 795 (1996).

[12] P. Cluzel et al., Science 271, 792 (1996).

[13] T. R. Strick, J. F. Allemand, D. Bensimon, and V. Croquette, Annu. Rev. Biophys. Biomolec. Struct. 29, 523 (2000).

[14] M. Elstner et al., Phys. Rev. B 58, 7260 (1998).

[15] P. Hohenberg and W. Kohn, Phys. Rev. B 136, B864 (1964).

[16] W. Kohn and L. J. Sham, Phys. Rev. 140, 1133 (1965).

[17] There exist early papers on computational modeling of simplified DNA structures or of small, finite piecies of DNA. Some early work of Ladik and collaborators, is presented in A. K. Bakhshi, P. Otto, J. Ladik, and M. Seel, Chem. Phys. 108, 215, (1986), and references therein.

[18] A. Lebrun and R. Lavery, Nucleic Acids Research 24, 2260 (1996).

[19] W. D. Cornell et al., J. Am. Chem. Soc. 117, 5179 (1995).

[20] A. Bensimon et al., Science 265, 2096 (1994).

[21] D. C. G. Klein et al., Appl. Phys. Lett. 78, 2396 (2001).

[22] M. W. Wu, L. P. Kouwenhoven, and L. L. Sohn (unpublished).

[23] J. F. Leger et al., Phys. Rev. Lett. 83, 1066 (1999).

[24] J. Jortner, M. Bixon, T. Langenbacher, and M. E. Michel-Beyerle, Proc. Natl. Acad. Sci. 95, 12759 (1998).

[25] P. T. Henderson et al., Proc. Natl. Acad. Sci. U. S. A. 96, 8353 (1999). 